\title{
A Miniaturized Ultra-Focal Magnetic Stimulator and Its Preliminary Application to the Peripheral Nervous System
}

\author{
Micol Colella, Micaela Liberti, Francesca Apollonio, \\ and Giorgio Bonmassar
}

\section{Introduction}

Transcranial magnetic stimulation (TMS) is a noninvasive brain stimulation technique that employs a high-intensity pulsed magnetic field sent through the scalp by a stimulating coil. According to Faraday's law, a time-varying magnetic field induces inside the brain tissue an electric field, which may elicit a neuronal response. Due to the lack of physical contact, TMS results in almost a painless stimulation compared to electric noninvasive techniques, and for this reason, it has been deeply investigated over the past decades. Many studies have demonstrated the effectiveness of TMS as a therapeutic solution for the treatment of different neuropsychiatric conditions, among which are major depression [1], chronic pain [2-5], epilepsy [6], and obsessive-compulsive disorder (OCD) [7]. Furthermore, TMS is extensively adopted in neuroscience research to investigate intracortical, cortico-cortical, and corticosubcortical interactions [6, 8, 9] and to assess causal relations between brain activity and behavior, as during speech [10-12] and motor mapping [13-19]. Despite the success, there are critical barriers to employing TMS in neuroscience research. One

\footnotetext{
M. Colella

Athinoula A. Martinos Center for Biomedical Imaging, Department of Radiology, Massachusetts General Hospital, Harvard Medical School, Boston, MA, USA

Department of Information Engineering, Electronics and Telecommunications (DIET), University of Rome "La Sapienza", Rome, Italy

M. Liberti · F. Apollonio

Department of Information Engineering, Electronics and Telecommunications (DIET),

University of Rome "La Sapienza", Rome, Italy

G. Bonmassar $(\bowtie)$

Athinoula A. Martinos Center for Biomedical Imaging, Department of Radiology,

Massachusetts General Hospital, Harvard Medical School, Boston, MA, USA

e-mail: giorgio.bonmassar@mgh.harvard.edu
} 
major restriction of TMS in its present form is its limited spatial resolution, which makes the task of focusing the stimulation exclusively to the targeted cortical region very challenging. The spatial resolution of TMS depends on the stimulating coil's geometry and dimensions. A deep investigation of the focality of the electric field induced by 50 TMS coils has been conducted by Deng et al. [20]. In this work, the authors showed that figure-of-eight coils are more focal than circular coils. For example, the Magstim $70 \mathrm{~mm}$ figure- 8 coil (P/N 9925, 3190) has a $15 \mathrm{~cm}^{2}$ focality $[20,21]$, while the Magstim $90 \mathrm{~mm}$ circular coil (P/N 3192) has a $70 \mathrm{~cm}^{2}$ focality $[20,21]$. Also, smaller coils are, in general, more focal than larger coils, with focality that can be as small as $5 \mathrm{~cm}^{2}$ [20]. For this reason, several attempts have been made to increase the spatial resolution of TMS by reducing the dimension of the stimulating coil [22-24]. To date, however, all the coils with reduced dimensions were designed for either invasive [24] or noninvasive animal applications [23]. In fact, as the dimension of the coil shrinks (diameter less than $2 \mathrm{~cm}$ ), larger electric currents (few hundreds of kiloamperes) are required to produce enough induced electric field in the human cortex $(\sim 60-100 \mathrm{~V} / \mathrm{m})$. Given the conventional engineering approach which uses a few turns $(<30)$ of wire winding inside an insulation housing, passing such large currents would produce excessive amounts of heat and large magnetic forces that may raise safety concerns or exceed specifications of conventional wires. To overcome such technological barriers, we have introduced flex circuit technology to design a new generation of miniaturized coils, which allows for a high number of turns (100 or greater) in a multilayer structure characterized by a reduced diameter $(15-20 \mathrm{~mm})$. Such an approach has been at first used to develop microscopic coils for invasive magnetic stimulation ( $\mu \mathrm{MS}$ ) capable of activating neuronal circuitry both in vitro [25] and in vivo [26]. Based on these results, a flex circuit technology was adapted to develop the first generation of miniaturized coils for noninvasive magnetic stimulation ( $\mu$ Coil). The concept behind the structure of the $\mu$ Coil consists of a certain number of parallel copper traces, named layers, deposited on a long flexible substrate wound in $N$ turns around a core. The prototype of $\mu$ Coil was built using a four-trace Kapton sheet, wound in 123 turns around a copper pin with a $1 \mathrm{~mm}$ diameter. Since each layer was $1 \mathrm{~mm}$ high, with a distance of $0.5 \mathrm{~mm}$ between two adjacent layers, we fabricated a single 8 -mm-tall circular solenoid ( $\mu$ Coil), with an outer diameter of $15 \mathrm{~mm}$. A figure-of-eight stimulator was obtained by pairing two single $\mu$ Coils, thus obtaining a structure with a maximum dimension of $30 \mathrm{~mm}$. To date, these coils are the only figure-of-eight coils capable of performing stimulation of the peripheral nervous system on healthy volunteers. In this chapter, we studied numerically the behavior of six different $\mu$ Coil geometries to show how each coil geometry affects the peripheral nerve stimulation threshold. 


\section{Models and Methods}

\section{$2.1 \quad \mu$ Coil Modeling}

The electromagnetic simulation software Sim4Life (v.5, Zurich MedTech, Zurich) was adopted in all of the simulations. A planar loop coil geometry represented the actual copper trace configuration. The space between two consecutive loops of $1.5 \mathrm{~mm}$ was selected to model the trace thickness and spacing. Six different geometries were considered, as shown in Fig. 1. The first geometry studied was a two-layered single circular coil, with each layer wound in 123 turns around a $1 \mathrm{~mm}$ air core. Second, two other layers, with the same characteristics as above, were added on top to make a four-layered single circular coil. The third geometry herein studied was the figure-of-eight configuration, which consisted of two four-layered single circular coils paired together and with currents circulating in the opposite phase. Three additional figure-of-eight coil models were built to study how the presence and length of an ideal iron core (i.e., linearity assumption: $\mathbf{B}=\mu_{\mathrm{r}} \mathbf{H}$ with $\mu_{\mathrm{r}}=60$ ) would modify the magnetic and electric field generated by the $\mu$ Coil. Each solenoid was wound in 100 turns and had an inner and outer diameter of $10 \mathrm{~mm}$ and $21 \mathrm{~mm}$, respectively. Three lengths of the iron core were considered: $9 \mathrm{~mm}, 23 \mathrm{~mm}$, and $41 \mathrm{~mm}$. Of all the geometries, the circular four-layered $\mu$ Coil and the figure-ofeight $\mu$ Coil were manufactured and tested. The iron-core $\mu$ Coils were studied to

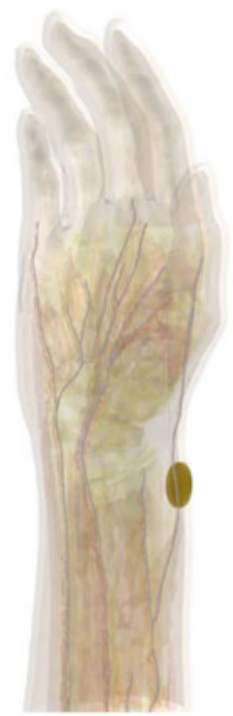

Circular- 2 Layers

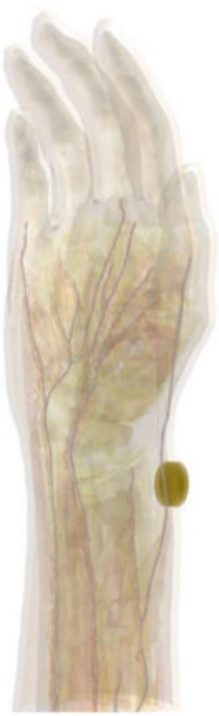

Circular-4 Layers

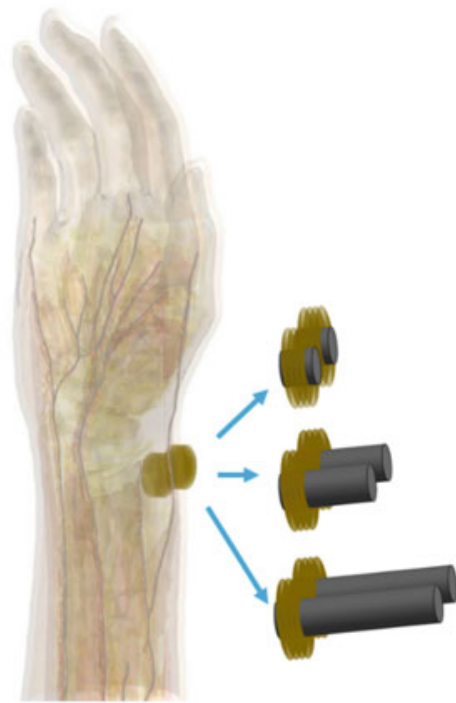

Figure-of-8

Fig. 1 Four figure-of-eight $\mu$ Coil models. $\mu$ Coil models and placement over the right arm of the neurofunctionalized model Yoon-Sun and the point where the two figure-of-eight $\mu$ Coils touch was precisely above the nerve trajectory in the wrist area 
enhance the magnetic field strength of the newly developed $\mu$ TMS coil for future cortical applications. The electromagnetic simulations were conducted using the Magneto Quasi-Static solver of the software Sim4life (v.5.9, Zurich MedTech, Zurich). This solver implements the quasi-static approximation that allowed decoupling the electric field from the magnetic field. Considering the source current density J, magnetic vector potential A was calculated from Ampere's law and used to compute the $\mathbf{B}$ and $\mathbf{E}$ field. EM simulations were performed by feeding a sinusoidal current with a $1.9 \mathrm{kHz}$ frequency in each $\mu$ Coil and a phase of $0^{\circ}$ in the first $\mu$ Coil and of $180^{\circ}$ in the second $\mu$ Coil when considering the figure-of-eight coil. The two coils were placed in a figure-of-eight coil over the arm of the anatomical, neurofunctionalized body model Yoon-Sun (ViP., v4 [27]), in correspondence of the superficial branch of the radial nerve, as shown in Fig. 1. The Yoon-Sun model is characterized by a detailed representation of the peripheral nervous system and the various nerve fibers. This simulation setup was validated by experiments conducted on healthy volunteers [28]. The $\mu$ Coil driving sinusoidal currents were set to $1 \mathrm{~A}$ for comparison among different configurations.

\subsection{Modeling Peripheral Nerve Stimulation: Titration Analysis}

To study the interaction between the induced electric field and the peripheral nervous system, the results of the EM simulations were fed into the dynamic Neuron solver version (v. 5.9) embedded in Sim4Life in a Multiphysics approach. The excitable behavior of the fibers was simulated using the McIntyre-Richardson-Grill model, which is based on a double-cable representation of the axon that allows separating electrical representations for the myelin and underlying internodal axolemma [29, 30].

Nerves can be excited by an external potential, $\mathrm{V}_{\text {ext, }}$ defined as in Eq. 1, where $\mathrm{C}$ is the linear path connecting each data point to an arbitrary zero voltage reference [31].

$$
\mathrm{V}_{\mathrm{ext}}=-\int_{C} \mathbf{E} \cdot d \boldsymbol{l}
$$

In this work, a titration analysis was conducted for all the simulated $\mu$ Coil geometries. Titration is the iterative process that allows determining the stimulation threshold of a nerve by stimulating it with pulses of increasing intensity. 


\section{Results}

\subsection{Magnetic Field Generated by the $\mu$ Coils}

Figure 2 shows the vector view of the $\mathbf{B}$ field generated by each $\mu$ Coil on the central cross-sectional plane. Inside the coil, the presence of more layers directs the $\mathbf{B}$ field along the axis of the coil. The magnetic field lines of forces diverge outside the coil and form closed loops. Doubling the number of layers increased the maximum intensity of $\mathbf{B}$ induced on the skin from 30 to $40 \mathrm{mT}$, thus by a factor of $33 \%$ without changing its direction. When forming the eight-shaped coil by pairing two circular $\mu$ Coils fed in counter phase, the $\mathbf{B}$ field lines formed a third loop at the center of the figure-of-eight coil, due to the coupling between the two coils. The use of the iron core modified the direction of the $\mathbf{B}$ field that was along the coil axis for the whole length of the core and parallel to the surface of the coil in the space between the two cores. At the interface between iron and air, vector $\mathbf{B}$ was deviated to respect the continuity of the normal component to the surface. Moreover, at the level of the nerve (i.e., $2.5 \mathrm{~mm}$ from the surface of the skin) $\mathbf{B}$ field intensity for the coil with the $41 \mathrm{~mm}$, long core is higher than that generated with the $23 \mathrm{~mm}$ long core and with the $9 \mathrm{~mm}$ long core, by $15 \%$ and $50 \%$, respectively.

a. Circular - 2 Layers

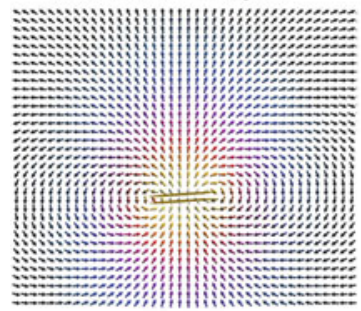

d. Figure-of-8 - Short core

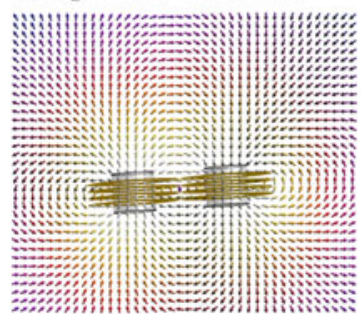

b. Circular - 4 Layers

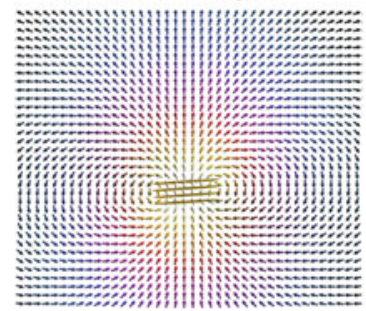

e. Figure-of-8 - Medium core

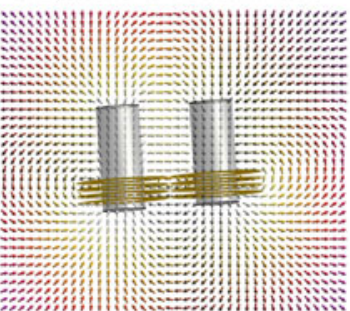

|B| [dB(40 mT)]

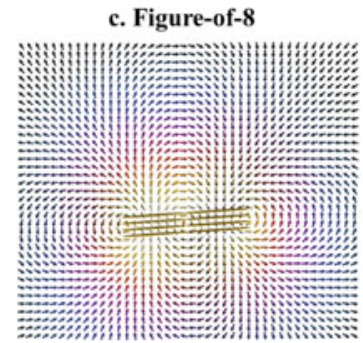

f. Figure-of-8 - Long core

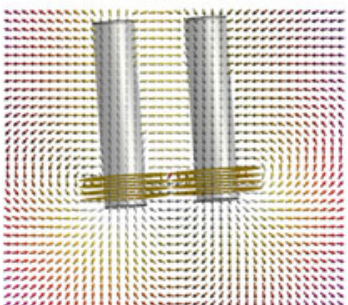

${ }_{0}$

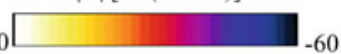

Fig. 2 Vector view of the $\mathbf{B}$ field generated by each $\mu$ Coil model. Arrows show direction, orientation, and intensity of $\mathbf{B}$. Intensities of $\mathbf{B}$ are represented by a color bar in a logarithmic scale with $0 \mathrm{~dB}$ referenced to $40 \mathrm{mT}$, which is the maximum $|\mathbf{B}|$ induced on the surface of the skin by the air core figure-of-eight $\mu$ Coil (Panel c) when fed with $1 \mathrm{~A}$ 


\subsection{Electric Field Induced by the $\mu$ Coils}

The single circular coil that was composed of four layers induced inside the tissues an electric field stronger than the field induced by the two-layered coil: the maximum $\mathbf{E}$ field induced at the level of the nerve with $1 \mathrm{~A}$ was $0.27 \mathrm{~V} / \mathrm{m}$ for the two-layered circular coil and $0.40 \mathrm{~V} / \mathrm{m}$ for the four-layered one (see Fig. 3, Panel a and b). The figure-of-eight coil configuration increased the intensity of the induced electric field on the nerve up to $0.6 \mathrm{~V} / \mathrm{m}$ (Fig. 3, panel C). Moreover, the direction of the $\mathbf{B}$ field (Fig. 2) induced an $\mathbf{E}$ field-oriented mostly along the longitudinal axis of the arm and (therefore) along the radial nerve. The presence of an iron core further oriented $\mathrm{E}$ along the nerve, as shown by the vector arrows in Fig. $3 \mathrm{~d}-\mathrm{f}$. Moreover, the longer the core, the higher was the maximum intensity of the induced $\mathbf{E}: 2.5 \mathrm{~V} / \mathrm{m}$ for $41 \mathrm{~mm}, 2.3 \mathrm{~V} / \mathrm{m}$ for $23 \mathrm{~mm}$, and $1.6 \mathrm{~V} / \mathrm{m}$ for $9 \mathrm{~mm}$. Figure 4 shows electric field components tangential $\left(\mathrm{E}_{\text {tan }}\right)$ and normal $\left(\mathrm{E}_{\text {norm }}\right)$ to the nerve fibers, for the two circular $\mu$ Coils and the air core figure-of-eight $\mu$ Coil. The figure-of-eight $\mu$ Coil induced a tangential component that was five and seven times higher than that induced by a four-layered and a two-layered single circular $\mu$ Coil, respectively, and a normal component that was two times lower.

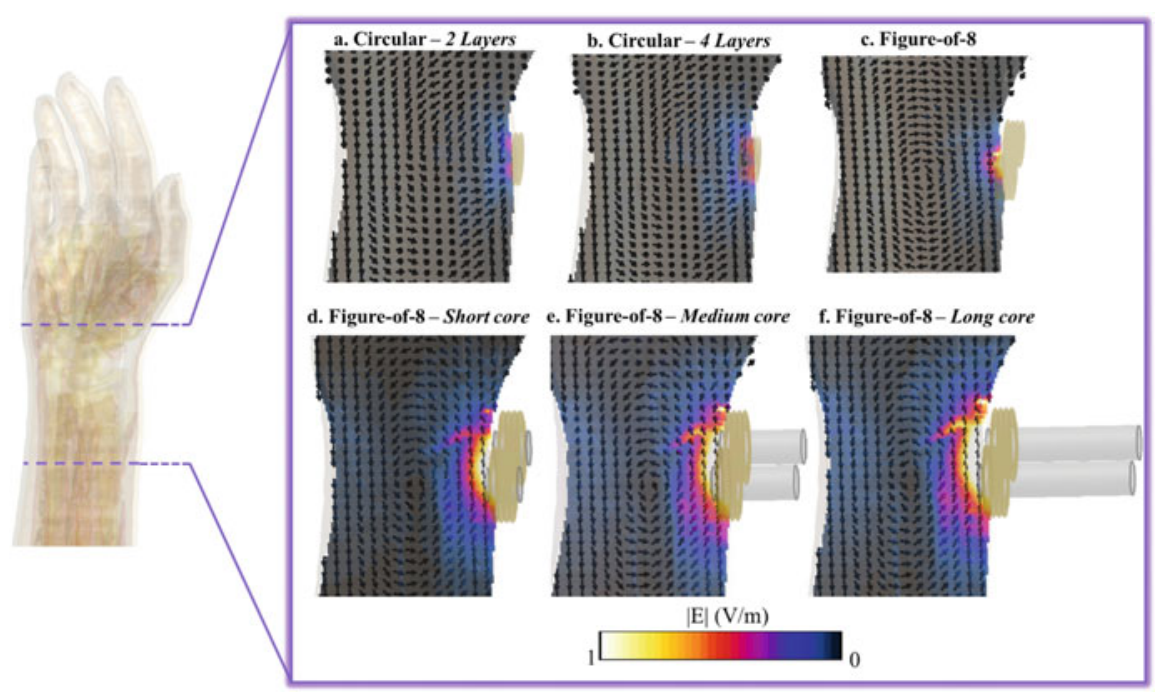

Fig. 3 Direction, orientation, and intensity of the $\mathbf{E}$ field induced in the arm of the Yoon-Sun model on the longitudinal plane of the arm that crosses the center of the stimulating coil. A vector view of the $\mathbf{E}$ field is superimposed on the intensities distribution map 

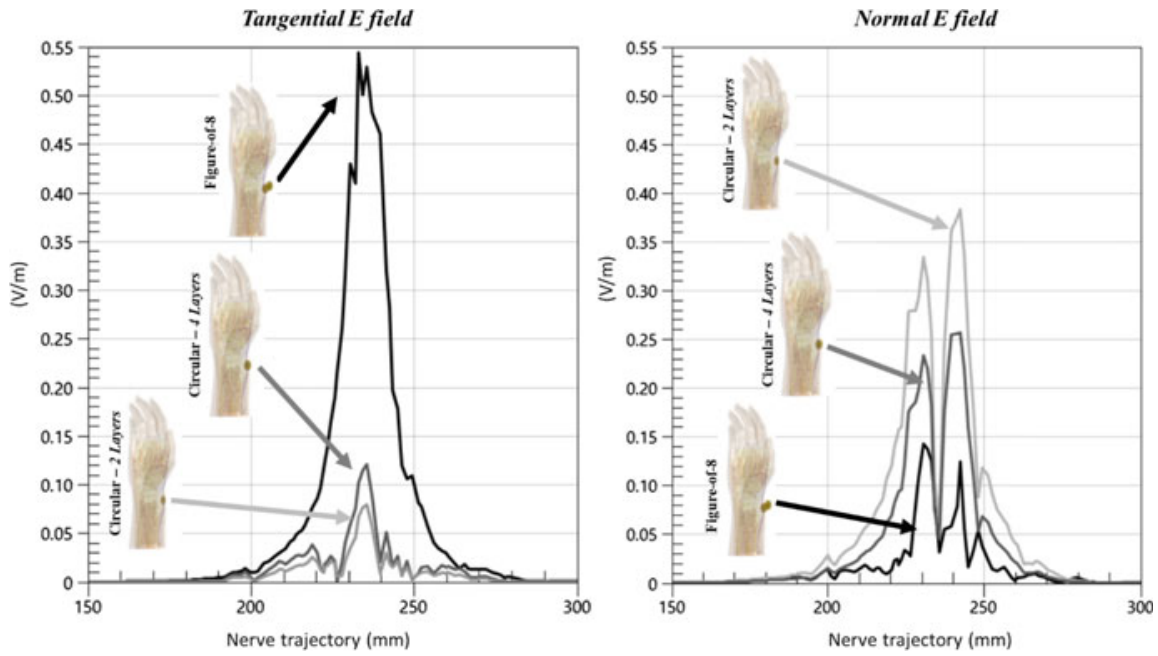

Fig. 4 The tangential (left) and normal (right) components of the $\mathbf{E}$ field-induced along the nerve by the two-layered circular $\mu$ Coil, the four-layered circular $\mu$ Coil, and figure-of-eight $\mu$ Coil

\subsection{Variation of the Peripheral Nerve Stimulation Threshold}

When simulating exposure of the arm to the two-layered single circular coil, the computed stimulation threshold for the radial nerve fibers varied between $259 \mathrm{~A}$ and 269 A. Nerve action potentials were generated near the extremities of the coil. Increasing the number of layers from two to four reduced the coil current threshold in each layer/coil to values between 177.56 A and 184.39 A, while the activation site of the neuronal response was not affected. The stimulation threshold was further reduced when simulating with a figure-of-eight coil: the five times higher induced tangential electric field of the figure-of-eight-coil reduced the threshold to values between $58 \mathrm{~A}$ and $58.5 \mathrm{~A}$; such a small range of variability may be due to the augmented focality. For all fibers, the activation site of the neuronal response was at the center of the figure-of-eight coil. Onset timing was not affected by the number of layers or by the change of coil shape and remained around an average time of $0.8 \mathrm{~ms}$. A reduction of the onset timing from 0.8 to $0.5 \mathrm{~ms}$, meaning an earlier spike, was obtained when changing from air to iron core. The presence of the core also reduced the stimulation threshold from $58 \mathrm{~A}$ to $14 \mathrm{~A}, 10.1 \mathrm{~A}$, and $9.4 \mathrm{~A}$ for the short, medium, and long core, respectively, and further oriented $\mathbf{E}$ field along the direction of the nerve. 


\section{Discussion and Conclusion}

The lack of focality of TMS stimulators is a significant barrier for the future deployment of this type of noninvasive neurostimulator in the clinics or neuroscience research. Several solutions have been proposed during the past years to overcome this limitation. For example, in 2010, Talebinejal and Musallam conducted a numerical study on a multilayer miniaturized coil made up of braided Litz wire [23] and demonstrated the feasibility of realizing a TMS coil reduced in size. Moreover, they showed that adding more layers increased magnetic field intensity while keeping the coil area constant. Following a similar geometry, in Tischler et al. 2011, a mini-coil with an outer diameter of $26.5 \mathrm{~mm}$ was developed. It is crucial to notice that all the attempts made to reduce dimensions of the TMS coils were conceived for either invasive [24] or noninvasive [23] animal models. To our knowledge, the newly developed figure-of-eight $\mu$ Coil, based on flex circuit technology [32], is the first prototype of miniaturized coil manufactured for on-human applications. Experiments conducted on the peripheral nervous system of healthy volunteers showed that the $\mu$ Coil could elicit somatosensory nerve action potentials (SNAPs) when the coil is placed over the superficial branch of the radial nerve. In this chapter, we conducted a numerical study that aimed to show how different possible $\mu$ Coil geometries can affect the radial nerve stimulation threshold. Six different topologies of $\mu$ Coils were modeled, based on the three different $\mu$ Coils actually manufactured: the single circular four-layered and the figure-of-eight coils were originally designed to be tested on the PNS, and the iron core figure-of-eight coil developed to target the cerebral cortex. Each coil was placed over the radial nerve superficial branch of the neurofunctionalized model Yoon-Sun [27]. A multiphysics approach was herein considered, and electromagnetic simulations were coupled with the dynamic neuronal solution. To study the effect of adding more layers, we first studied a two-layered single circular $\mu$ Coil and compared it with the four-layered one. The results showed that increasing the number of layers from two to four increased the intensity of the $\mathbf{B}$ field by $50 \%$, thus inducing a stronger $\mathbf{E}$ field. As a consequence, the stimulation threshold was lower for the four-layered coil. A figure-of-eight coil was obtained by pairing together two four-layered circular coils. Such $\mu$ Coil was able to generate a stronger magnetic field, thus inducing a stronger electric field on the nerve. Moreover, the figure-of-eight coil oriented the $\mathbf{E}$ field along the trajectory of the nerve fibers, leading to a better coupling between the coil and the nerve and a lower stimulation threshold. This coupling is further enhanced by addition of an iron core. Additionally, the results showed that the presence of the core not only increased intensity of the induced electric field, as expected, but also reduced the onset timing of the first spike. Nevertheless, it should be noted that saturation of the iron core was not considered in this study. Thus, computed stimulation threshold was underestimated, due to the overestimation of both $\mathbf{B}$ and $\mathbf{E}$ field intensities. However, we are planning to model next the nonlinearities of a real core. We confirmed that the figure-of-eight coil was more efficient than the circular coil as it enhanced the focality and the coupling between nerve fibers and $\mathbf{E}$ field. Moreover, the results showed that the presence of 
an iron core further reduced stimulation threshold while increasing depth, making this geometry suitable for investigating the $\mu$ Coil stimulation of the brain cortex.

\section{References}

1. Pascual-Leone, A., Walsh, V., \& Rothwell, J. (2000). Transcranial magnetic stimulation in cognitive neuroscience - virtual lesion, chronometry, and functional connectivity. Current Opinion in Neurobiology, 10(2), 232-237.

2. Kobayashi, M., \& Pascual-Leone, A. (2003). Basic principles of magnetic stimulation. Lancet, 2, 145-156.

3. Fitzgerald, P. B., Fountain, S., \& Daskalakis, Z. J. (2006). A comprehensive review of the effects of rTMS on motor cortical excitability and inhibition. Clinical Neurophysiology, 117 (12), 2584-2596.

4. Najib, U., Bashir, S., Edwards, D., Rotenberg, A., \& Pascual-Leone, A. (2011). Transcranial brain stimulation: Clinical applications and future directions. Neurosurgery Clinics of North America, 22(2), 233-251.

5. Valero-Cabré, A., Pascual-Leone, A., \& Coubard, O. A. (2011). Transcranial magnetic stimulation (TMS) in basic and clinical neuroscience research. Revue Neurologique (Paris), 167(4), 291-316.

6. Rotenberg, A. (2010). Prospects for clinical applications of transcranial magnetic stimulation and real-time EEG in epilepsy. Brain Topography, 22(4), 257-266.

7. Carmi, L., Alyagon, U., Barnea-Ygael, N., Zohar, J., Dar, R., \& Zangen, A. (2018). Clinical and electrophysiological outcomes of deep TMS over the medial prefrontal and anterior cingulate cortices in OCD patients (Vol. 11, p. 158). Brain Stimulation.

8. Horvath, J. C., Perez, J. M., Forrow, L., Fregni, F., \& Pascual-Leone, A. (2011). Transcranial magnetic stimulation: A historical evaluation and future prognosis of therapeutically relevant ethical concerns. Journal of Medical Ethics, 37(3), 137-143.

9. Rotenberg, A., Horvath, J., \& Pascual-Leone, A.. (2014). Transcranial magnetic stimulation series editor.

10. Jennum, P., Friberg, L., Fuglsang-Frederiksen, A., \& Dam, M. (1994). Speech localization using repetitive transcranial magnetic stimulation. Neurology, 44(2), 269.

11. Wassermann, E. M., et al. (1999). Repetitive transcranial magnetic stimulation of the dominant hemisphere can disrupt visual naming in temporal lobe epilepsy patients. Neuropsychologia, 37, 537-544.

12. Epstein, C. M., et al. (1999). Localization and characterization of speech arrest during transcranial magnetic stimulation. Clinical Neurophysiology, 110, 1073.

13. Wassermann, E. M., McShane, L. M., Hallett, M., \& Cohen, L. G. (1992). Noninvasive mapping of muscle representations in human motor cortex. Electroencephalography and Clinical Neurophysiology/Evoked Potentials, 85(1), 1-8.

14. Brasil-Neto, J. P., Cohen, L. G., Panizza, M., Nilsson, J., Roth, B. J., \& Hallett, M. (1992). Optimal focal transcranial magnetic activation of the human motor cortex: Effects of coil orientation, shape of the induced current pulse, and stimulus intensity. Journal of Clinical Neurophysiology, 9(1), 132-136.

15. Cincotta, M., et al. (2006). Mechanisms underlying mirror movements in Parkinson's disease: A transcranial magnetic stimulation study. Movement Disorders, 21(7), 1019-1025.

16. Cincotta, M., \& Ziemann, U. (2008). Neurophysiology of unimanual motor control and mirror movements. Clinical Neurophysiology, 119, 744.

17. Civardi, C., Vicentini, R., Collini, A., Boccagni, C., Cantello, R., \& Monaco, F. (2009). Motor cortical organization in an adult with hemimegalencephaly and late onset epilepsy (Vol. 460, p. 126). Neuroscience Letters. 
18. Martinez, M., Brezun, J. M., Zennou-Azogui, Y., Baril, N., \& Xerri, C. (2009). Sensorimotor training promotes functional recovery and somatosensory cortical map reactivation following cervical spinal cord injury. The European Journal of Neuroscience, 30(12), 2356-2367.

19. Karl, A., Birbaumer, N., Lutzenberger, W., Cohen, L. G., \& Flor, H. (2001). Reorganization of motor and somatosensory cortex in upper extremity amputees with phantom limb pain. The Journal of Neuroscience, 21(10), 3609-3618.

20. De Deng, Z., Lisanby, S. H., \& Peterchev, A. V. (2013). Electric field depth-focality tradeoff in transcranial magnetic stimulation: Simulation comparison of 50 coil designs. Brain Stimulation, $6(1), 1-13$.

21. Hovey, C., \& Jalinous, R. (2006). The guide to magnetic stimulation. Magstim, 20(4), 284-287.

22. Salinas, F. S., Lancaster, J. L., \& Fox, P. T. (2009). 3D modeling of the total electric field induced by transcranial magnetic stimulation using the boundary element method. Physics in Medicine and Biology, 54, 3631.

23. Talebinejad, M., \& Musallam, S. (2010). Effects of TMS coil geometry on stimulation specificity. In 2010 Annual international conference of the IEEE Engineering in Medicine and Biology Society EMBC'10, pp. 1507-1510.

24. Tischler, H., et al. (2011). Mini-coil for magnetic stimulation in the behaving primate. Journal of Neuroscience Methods, 194(2), 242-251.

25. Bonmassar, G., Lee, S. W., Freeman, D. K., Polasek, M., Fried, S. I., \& Gale, J. T. (2012). Microscopic magnetic stimulation of neural tissue. Nature Communications, 3, 910-921.

26. Park, H. J., Bonmassar, G., Kaltenbach, J. A., Machado, A. G., Manzoor, N. F., \& Gale, J. T. (2013). Activation of the central nervous system induced by micro-magnetic stimulation. Nature Communications, 4, 1-9.

27. Gosselin, M.-C., et al. (2014). Development of a new generation of high-resolution anatomical models for medical device evaluation: The virtual population 3.0. Physics in Medicine and Biology, 59(18), 5287-5303.

28. Colella, M., et al. (2019). A microTMS system for peripheral nerve stimulation. Brain Stimulation, 12(2), 521.

29. McIntyre, C. C., \& Grill, W. M. (2002). Extracellular stimulation of central neurons: Influence of stimulus waveform and frequency on neuronal output. Journal of Neurophysiology, 88(4), $1592-1604$.

30. McIntyre, C. C., Richardson, A. G., \& Grill, W. M. (2002). Modeling the excitability of mammalian nerve fibers: Influence of afterpotentials on the recovery cycle. Journal of Neurophysiology, 87(2), 995-1006.

31. Sim4life Documentation. ZMT Zurich MedTech AG.

32. Colella, M., et al. (2019). Ultra-focal magnetic stimulation using a $\mu$ TMS coil: A computational study, 2019. In 41st Annual international conference of the IEEE Engineering in Medicine and Biology Society, pp. 3987-3990

Open Access This chapter is licensed under the terms of the Creative Commons Attribution 4.0 International License (http://creativecommons.org/licenses/by/4.0/), which permits use, sharing, adaptation, distribution and reproduction in any medium or format, as long as you give appropriate credit to the original author(s) and the source, provide a link to the Creative Commons license and indicate if changes were made.

The images or other third party material in this chapter are included in the chapter's Creative Commons license, unless indicated otherwise in a credit line to the material. If material is not included in the chapter's Creative Commons license and your intended use is not permitted by statutory regulation or exceeds the permitted use, you will need to obtain permission directly from the copyright holder.

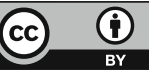

